\title{
Developing a Cornerstone "Human in the System" Engineering Design Course
}

\author{
F.A. Salustri and W.P. Neumann \\ Department of Mechanical and Industrial Engineering \\ Ryerson University
}

Abstract: The authors describe their experiences creating a cornerstone engineering design course for mechanical and industrial engineering undergraduate students. Starting with a tabula rasa, we have been working to create a one-semester design experience that integrates Human Factors (HF) directly into every aspect of engineering design. In the last decade, we have identified three key issues with which we grapple: lack of integration of HF in design; lack of access to cohesive HF data; and dysfunctional student teams. Given the lack of available information upon which to draw for the design of this course, we adopted a CQI-like iterative, organic, and evolutionary approach. In this paper, we present many of the ways we have attempted to address these issues, relating to courseware development, course management, assessment and grading, and student and instructor support. We summarize by presenting our advice to others who are looking to fully embed HF or other non-design fields into a cogent design experience for their students. All our courseware and tools are available freely on the web.

\section{Introduction}

Ten years ago, the Department of Mechanical and Industrial Engineering at Ryerson University decided that a new cornerstone undergraduate design course was needed for both Industrial and Mechanical students. The course was conceived of to address two problems that the Department had noted:

- Industrial engineering students were receiving virtually no instruction in engineering design, which hampered the execution of their Capstone projects as well as other, smaller design-based projects in other courses; and

- Mechanical engineering students were receiving virtually no instruction in the impact their designs had on human users (including not only end-users, but manufacturers, maintenance personnel, etc.).

By focusing on "product engineering design", the course would integrate design and Human Factors (HF) into a single course. That course, MEC325, Introduction to Engineering Design, has been taught by the authors since its inception.

Our guiding principle has been that a product's interaction with human users, throughout the product's life, is the most fundamental driver of design decisions. The essential question driving our development of this course is: How can we create an integrated and cohesive course blending both design and HF to fit in one semester? This paper will describe three major problems that we encountered over the last decade of trying to answer this question, and the solutions we have implemented (successfully and otherwise) to manage them.

Since the problems we have encountered are coupled to each other, we will describe all the 
problems first, then present the various solutions. Finally, we will discuss our intentions for the future and summarize our work to date.

Our review of existing courses, textbooks, and approaches informed us that no suitable pedagogic framework or method exists to address our Department's needs. We therefore fell back on our own knowledge of Continuous Quality Improvement (CQI) [1], and applied its principles to the development of MEC325. Specifically, each year, the instructors kept notes on identified shortcomings in the course; then, between offerings, we would reflect on those shortcomings, identify reasonable approaches to resolving a few of them, and implement them in subsequent offerings. Those resolutions that seemed to improve some aspect of the course were kept; others were abandoned or replaced. Thus, the development of the course has been largely organic, nonlinear, and evolutionary, rather than strictly managed, directed, and linear. We note that this approach has also allowed the instructors to remain flexible and responsive to both emerging opportunities and organizational disturbances.

\section{Description of Problems}

Each year that the authors have taught MEC325, different issues in course delivery and student performance have manifested. We address one issue in one year, only to have a different and often unrelated issue emerge the next year. Over the years, the authors have found that all these issues seem to stem from three significant issues in deploying a meaningful student experience that covers both design and HF in a cohesive way. There are also a number of aggravating factors that play into those problems. These are outlined below.

These problems can be generalized to other fields of engineering, such as sustainability, that ought to be inherently part of designing even at the introductory level. We only address HF here, but we hope our efforts will inspire others working at the boundaries between design methodology and other design affected fields.

We note here that all the courseware discussed in this paper, in its current incarnation at least, is available via Salustri's wiki for MEC325.

\section{Problem 1: Lack of Integration of Design and HF}

MEC325 is intended to provide a one-semester learning experience in both design and HF. Combining these two disciplines into a cohesive whole is our most fundamental problem.

The authors reviewed all the major readily-available english-language design textbooks and design-oriented and education-oriented conferences. We remain unaware of any other design courses that genuinely include HF as a driving force underlying design. Textbooks may cover $\mathrm{HF}$, but the subject is invariably treated as a "sidecar" issue to efficiency and cost. HF is not integrated into any of the typical design methodologies for introductory courses. Safety, while important, is only one aspect of HF, and is generally regarded as a way to manage product failures rather than preventing failures by attending to human capabilities and limitations during design. These pedagogical treatments of HF also fail to see HF knowledge as a means to 
achieving excellence in design that furthers the performance agenda in design projects. Furthermore, the authors found no clear model on how such a course might be delivered in one semester.

\section{Problem 2: Lack of Access to Cohesive HF Data}

As we grappled with the problem of integrating HF and design, we became aware of the inherent difficulty in finding usable, understandable HF information for 2nd year students.

There are many readily accessible sources of HF data. However, they are often inconsistent with one another and difficult to apply to specific practical cases that occur in MEC325 design projects. For instance, consider the task of carrying the jar of a kitchen blender from a kitchen counter to a dining room table. HFs involved in this task include the strength of the fingers, wrist, and arm; hand-to-eye coordination; stability of grip; steadiness during walking, visually estimating distances; etc. A blender design that fails on any of these HFs could lead to usage failures and even injury. It is simply not possible to find data easily on all these measures that relate directly to lifting and carrying weights such as blenders. Existing data sources require a reasonable preexisting knowledge of HF and are thus impenetrable to MEC325 students. We have noticed students tend to spend too much time trying to find and understand HF data, and not enough time using that data to create quality designs.

Furthermore, the lack of easily accessible information and the workload involved in finding and using it led many teams to make claims about human users that were not only obviously incorrect but often bordering on the discriminatory. An unfortunately typical example of this was a claim by a team that "Pregnant women cannot change automobile tires" (emphasis added by authors).

\section{Problem 3: Dysfunctional Teams}

MEC325 currently has between 60 and 70 student teams per year, with typically 5 students per team. Our experience is that, if left unchecked, as many as one quarter of teams experience significant dysfunction. Typical behaviours included marginalizing team members, ignoring teammates' contributions, removing teammates' work from the project reports for fear their own marks would suffer, and even abusive language and behaviour. Another aspect of this problem is the preconceptions of the students: Industrial Engineering students are not very interested in design, and that Mechanical Engineering students are not very interested in HF - and yet they must work together in teams. This results in very poor performance on final projects, and a tremendous workload for instructors who must remediate those teams to ensure fair assessment of all students.

However, education in teamwork is not covered in, or before, MEC325. At this time, the authors are neither able to influence prerequisite courses nor substantively alter MEC325 to introduce learning about teamwork. We therefore need to provide a team-based experience while also making grading as independent as possible of team dysfunction. 


\section{Additional Aggravating Factors}

The three problems noted above are aggravated by a number of factors:

- MEC325 is a large class of about 300 students, and since there are very few suitably trained graduate students in our department, attending the 27 contact hours per week for this course falls principally on the instructors.

- Our 2nd year students lack the engineering background to analyze and iterate on their designs in detail.

- Our students tend to be distracted by the format of the deliverables we expect of them and produce technically weak, but beautifully rendered, content.

- At the end of the semester, the instructors typically have no more than 14 days to grade $60-70$ project reports, often over 200 pages long each, 300 design journals, and 300 final exams; this workload significantly increases the odds of grading errors and oversights.

\section{Attempted Resolution Approaches}

In this section, we will review various techniques and tools that we have introduced to address the problems and their aggravating factors. Not all our attempts have been successful, resulting in an ongoing, organic evolution of the course as we respond on a yearly basis to emerging aspects of the problems - consistent with our CQI-like approach to course development.

\section{Approaches for Problem 1: Content Integration}

Ten years ago, when the course was first created, the instructors had no idea how to integrate the material because we could not find any appropriate reference materials. It was, to the best of our knowledge, the first such course in Canada. While there are some engineering programs in other countries (e.g., TU Delft's Industrial Design Engineering program) that incorporated HF into engineering, those programs are so different from Canadian programs that meaningful transfer of practice was problematic.

We therefore taught the first offering of MEC325 as two discrete but overlapping courses. Neumann taught lectures on HF and Salustri taught lectures in design. We interleaved our lectures so as to accommodate the timing of various assignments and projects, but not with respect to the nature of the material being presented.

To help coordinate activities, both instructors attended all lectures even though only one of us taught on any given day. An unintended side-effect of this was that the instructors began to recognize in vivo significant areas of overlap between design and HF issues, especially in requirements elicitation and concept evaluation. The discovery of these synergies became key areas in which we have developed over time novel courseware specifically targeted at the ubiquitous embedding of HF in design. Furthermore, the ability for the "inactive" instructor in any given lecture to still interject relevant comments became a key tactic to demonstrate to students the underlying unity of HF and design.

This became the foundation of our CQI loop, and the source of key observations that we would 
leverage into opportunities for course improvement.

The "tag team" practice of attending each other's lectures remains a key feature of our pedagogy today. In the post-COVID epoch of remote/online teaching, this approach yields further benefits by having an "extra pair of eyes" to track chat messages and "raised hands" in Zoom so that the instructor who is lecturing can focus more on the cogent presentation of material.

Every year after the course's first offering, during the summer, we would review the past year's offering for unexploited synergies, and try to redevelop the corresponding course elements to reflect those synergies.

An obvious example is the alignment of terminology between HF and design, such as the difference between "function" and "behaviour" in the two fields. While these are not difficult problems to resolve, it can be onerous to do so because it requires substantive review of course notes, slides, and other documentation, as well as overcoming habits the instructors had formed in their use of discipline-specific language. Still, even just using the same language reduced the friction in communicating with students, and promoted deeper discussion with and among them, by creating more uniform mental models: students worried less about what individual concepts meant in "design" vs "HF" terms, and more about the application of those concepts to their design projects.

Another general strategy we have used repeatedly involves modifying known tools and methods from the design literature over the years, to incorporate HF and better fit the needs of our students. One example of this is a Usage Scenario (US) diagram, which we use to represent diagrammatically the tasks necessary for a user to achieve a goal with a product. This diagram type originated from the work of Stone, Wood, and Crawford [2] in reverse engineering and product modularization. However, over the last two decades, and through several iterations, we have turned the diagram into something substantially different. US diagrams are now primarily used by teams to (a) lay out the "operational flow" of their designs, and (b) identify key tasks users must perform that will form HF bottlenecks - tasks that will substantially limit the inclusivity of the design by placing demands on vulnerable users in excess of their capacity. In keeping with our CQI approach, each year we look for problems students experienced in understanding, constructing, and using USs, and then attempt to refine the format and nature of the diagrams for subsequent course offerings. Examples of some of these refinements are described in subsequent sections.

Another design tool that we have adapted is the weighted decision matrix (WDM) for design concept evaluation. Originally conceived for engineering by Stuart Pugh [3] as a qualitative tool, we found our students needed the familiarity of a quantified tool to guide their decisions. For many years, we expected each team to collaboratively build a single WDM containing all design concepts the team generated, using a modified pairwise comparison method to establish weights for the various criteria in the WDM. However, over the years we noticed four significant problems with this approach: (1) team dynamics would inappropriately influence ratings decisions; (2) teams could "reverse engineer" WDMs based on what they had subjectively decided was the "best" design; (3) concept ratings were rarely grounded in HF considerations; 
and (4) our 2nd Year students' lack of knowledge of understanding about manufacturing, sustainability, and other aspects of design, prevented them from executing justifiable pairwise comparisons of criteria weights. The technique seemed too advanced for an introductory course.

To address this, we have recently revised the entire concept evaluation task. Firstly, we removed pairwise comparison from the task entirely to obviate problems arising from students' lack of engineering knowledge. All criteria in our new decision matrices (DM) are now of equal weight. This also implied a change in attention to safety. Previously, safety issues were often seen to have the highest priority in decision making. By shifting safety to be a constraint, instead of a design criterion, it became possible to lock in attention to safety, while allowing the use of a simplified, equal-weight DM.

The second change depends on changes to the use of Personas (described in detail in the next section): each student must now conduct an individual concept evaluation of all concepts with a focus on the Persona for which they are responsible; thereafter, team members combine the individual DMs by straight superposition - thus eliminating a major source of "political" influence between team members, while also making more obvious the need to "think of, and advocate for, the user" when evaluating potential design concepts, and also making their own subjective preferences harder to hide.

Similarly, we have also sought to customize common tools and methods in HF to fit with our overall design process in MEC325.

Hierarchical Task Analysis (HTA) [4] is commonly used in HF to break down tasks into subtasks for the sake of identifying the specific tasks and actions that human users will have to perform with a product (or in a job) to achieve a goal. In 2017, we introduced HTA as an early component of the design projects, in which teams would develop a partial HTA of their reference design, focusing on the usage life-cycle stage. (Our 2nd year students could not be expected to develop HTAs for, say, product manufacturing or end-of-life processing due to lack of background knowledge and training.) Our expectation was that performing HTAs for their reference designs would help them overcome two specific problems we had observed in Usage Scenarios in previous years: (1) that the USs were either too coarse or too detailed to be useful in making design decisions, and (2) that students typically overlooked the necessary steps of setting up a product for use and then putting it away after use, both of which can lead to substantial HF related problems.

However, even with clear deliverables, and coverage in both lectures and studios, students struggled to develop adequate HTAs, which only delayed their work on other project components. Furthermore, students complained that HTAs and USs seemed to be conceptual duplicates of each other, which they viewed as an unnecessary redundancy. Thus, in 2019, we eliminated explicit HTAs from the course. Instead, we embedded one of their key aspects - that every major task includes setup, usage, and "put-away" stages that must be clearly identified in their USs. We revised the general layout of USs to help make evident to students that these three stages are necessary, and also to facilitate grading deliverables. Furthermore, we require students to explicitly address each of the three stages in their decision-making and justifications. 
We recognize that we may simply have not implemented HTA properly for our course; thus, if we find the opportunity in the future, however, we may attempt to integrate more of the HTA method into the courseware. This highlights another important aspect of our CQI approach to course development: failed solutions may have failed due to our own shortcomings in developing the methods, tools, and courseware. The instructors are themselves elements in the teaching and learning system that is MEC325, and we must remain attentive to our own self-created problems.

Over the last few years, we have been developing the concept of Personas as a mechanism to connect HF to engineering requirements. Personas are hypothetical, archetypal users intended to help designers visualize and empathize with their users. More details of our work with Personas is given in the next section.

Furthermore, we refine team project design briefs annually to keep students away from types of solutions we know are beyond their abilities and of consistent complexity with other projects. An example of this is in our project on Maritime Escape/Life-Support Systems. When originally introduced several years ago, teams would very often design large and complex solutions such as the Viking Norsafe JYN-75. Such attempts would fail disastrously because our students simply lack the skills or time to execute such a design. Many of them believed (incorrectly) that the more complex the design, the higher their grade.

To address this, we began reviewing projects yearly, and altering project descriptions in ways that we expected would drive students to more sensible designs if they were diligent in following instruction. The current version of this project's design brief includes many "hints" intended to keep students away from designs that we know they would not be able to complete.

Finally, we have started using 4th year undergraduate students as teaching assistants. Having taken the course themselves, they are far more able to assist in running studios and labs than typical graduate students who do not have this training. This also helps manage the workload the course induces on the teaching team generally.

\section{Approaches for Problem 2: HF Information}

We realized relatively early in MEC325's development that we had to find a way to connect HF concerns to engineering requirements. For instance, setting an engineering requirement of a maximum weight on a domestic-use ladder could be directly tied to the strength capabilities of the targeted users.

To that end, we have over the last few years started using Personas. In the first few years, Personas were described simply with a short paragraph of text. Lecture material provided a list of characteristics of any "good" Persona description, and several examples were given. Each student was expected to create and design for five different Personas. A given team was therefore typically responsible for around 25 Personas.

However, over the following years we observed that students had a very difficult time 
developing meaningful Personas. Many Personas were just minor variations of others. Most disturbingly, this free-text approach to Personas led many students to make statements about their users that could easily be seen as exclusionary of many different abilities - if not decidedly discriminatory.

The extent of this problem was brought to our attention in 2017 by one of our 4th year TAs, who we subsequently hired to develop courseware specifically intended to address issues of inclusivity and diversity. That courseware was reported at CEEA [5] and is freely available on the web, and material for instructors and TAs is available on request. The package includes modules about Personas, designing for the elderly and for women, as well as general principles for designing for inclusivity; there are also modules covering the role of engineering in society and touching on a number of ethical issues.

Concurrently, we changed the description of Personas from free-form text to a chart intended to highlight specific HF capabilities for each persona and discourage teams from making the kind of discriminatory remarks that have no place in engineering design. Most importantly, we reduced the number of Personas per team. As of 2018, each team member would develop and "be responsible for" only one Persona. This had the practical impact of reducing student (and grader) workload. We also stressed that each student had to "design for" their Persona and make sure their Persona was safe, satisfied, and respected by their ultimate design.

The new material was deployed in Fall 2018, and remains a key part of the course. While students paid more attention to the HFs of Personas - e.g., Personas were more often mentioned as justification for various engineering requirements and design decisions - the Personas were still too similar to one another. It was at this point (early 2019) that we realized we were simply expecting too much of our students with respect to finding and understanding HF information.

Having found no reasonable, usable HF data resources, we have begun to develop our own. With the continued financial support of Ryerson's Centre for Excellence in Learning and Teaching, we hired another undergraduate research assistant to help us find and organize data relevant to the kinds of projects typically assigned in MEC325 over the summer of 2019.

As we began collecting usable HF data, we noticed that there was virtually no procedural information available that could help 2nd year students apply the HF data to improve their designs. The research assistant was then asked to develop methods for students to use. Based on our review of the literature, we decided to focus on only three HFs - vision, hearing, and strength - to ensure we would have complete coverage in at least some HFs by Fall 2019. We developed a "default" qualitative, and admittedly less reliable, way of assessing other HFs (e.g., memory, smell, etc.). The modules provide students with links to reliable HF data sources, and worked examples developed by the research assistant of how to use the data to estimate the fraction of a population excluded from using a particular product based on the HF demands imposed on users by the product. This material is not perfect, and further development is being considered. In keeping with our CQI approach, we will observe how students respond to the material and how the quality of their designs changes; armed with that information, we will determine how to best improve these modules. 
To provide further guidance to students, and in keeping with another principle of our course that designing is about changing how things are to how we wish they were - we separated the HF analysis into two stages. The first stage requires teams to determine the HF demands placed by their reference design on its users. (Example: " $15 \%$ of the population lacks the vision capabilities to operate the reference design") The methods we provide let them describe those demands in terms of the percentage of potential users who cannot use the reference design due to HF concerns. We believe this helps cement in students' minds the impact of design decisions on "many real people". Teams then expand the range of inclusivity according to their product strategy. (Example: "We want no more than $2 \%$ of the population to be excluded from using our product due to their vision capabilities.") Given a larger population to be included in the new design and their HF capabilities, they now reverse the calculations, moving from inclusivity measures to the range of the various HFs to which they must design to achieve that level of inclusivity. (Example: "All labels must be printed in 16pt font or larger.")

The HF capabilities defined by each team are also used to define their Personas, such that each Persona has at least one or two HF capabilities that are at the edge of the HF envelope marking the extent of the included population. Per the example in the preceding paragraph, for example, a team might have one or two Personas with 20/100 vision (which marks the approximate $2 \%$ boundary of the population) and all their other Personas would have average or even above average vision.

This material was deployed in Fall 2019 and is freely available on the web. We hope to present this work at the 2021 CEEA Conference.

According to course exit surveys, all these modules are well received by students. In grading the reports, we noticed significantly fewer "silly mistakes" arising from neglect of HFs; however, deeper problems arising the broader implications of HF capabilities remain problematic. We continue to work on this aspect of the course, to help streamline the application of HF in this introductory context.

The single most common complaint was with the difficulty in treating strength. This remains problematic due to the inherent complexity of physical strength and the inconsistency of presentation between various data sources. For instance, driving a screw horizontally into wood at chest height involves muscles in the fingers, hands, lower and upper arms, shoulders, back, and legs. We expect to begin treating various cognitive HFs next, such as memory and reasoning in the coming years.

\section{Approaches for Problem 3: Addressing Team Dysfunction}

Our goal here is to minimize team dysfunction, or at least identify it early enough to allow fast and thorough intervention. Besides the pragmatic concerns raised earlier in this paper, our approach is to make evident to students that they are part of the "system" in which new products are made, used and abused, and eventually discarded. As such they must become aware of their role in providing their teammates as well as their users with a beneficial design experience. 
The problem of student interest based on their program is, we believe, addressed if we can address Problems 1 and 2 (above). Thus, in this section, we focus on other aspects of team dysfunction as we have observed it in MEC325.

Firstly, Salustri developed an algorithmic approach to team formation in the 1990s based on Jungian personality types. Anecdotal evidence collected by Salustri suggests that team dysfunction is significantly lower when the algorithm is used to form teams than any other method we have tried. Typically, when the algorithm is used, we estimate $2 \%-5 \%$ of teams exhibit dysfunctional behaviours requiring intervention; but when the algorithm is not used, we have estimated as many as $20 \%-30 \%$ of teams are dysfunctional. We are not sure whether the algorithm actually works, or whether its use simply induces a self-fulfilling prophecy in students ("the computer told me I would get along with my teammates," so they actually do get along). We have lacked the resources to properly assess the underlying effects; however, we remain committed to the algorithmic formation of teams for now.

Secondly, we require all students to "claim responsibility" for standardized elements of their project on a 0-4 scale, on a Workload Distribution Form (WDF). We have tried several different measures of students' contributions over the years: hours spent, "effort", "value" as determined collaboratively by the team, page count, etc. So far, "responsibility" seems the most effective in that it is the concept most easily understood by students and that causes the least argument among team members. We have found that teams with highly variable results on WDFs tend to be highly dysfunctional.

A special spreadsheet developed by Salustri over several years allows reports to be graded by project component as if one person wrote it. The WDF data is then used by the spreadsheet to calculate individual marks for each student. The idea is that a student claiming the most responsibility for a project component should benefit or suffer the most, depending on whether that component was well executed. The range of marks produced by the sheet for a given team is typically $+/-10 \%$ around the report's grade. Allowances are made by the spreadsheet to accommodate for variable team sizes, and any penalties (e.g., for lateness) imposed by the instructors. The use of this sheet greatly expedites grading at the end of the semester, when the instructors are under great pressure to calculate and submit final course grades, while helping ensure fair and consistent assignment of individual grades.

Thirdly, as of Fall 2019, we have begun to use ITPmetrics for team peer reviews, and use the data generated from its surveys to identify dysfunctional teams. ITPmetrics uses surveys to assess various metrics of team performance and cohesion. We have found the reports it produces quite useful to guide discussions with teams. Furthermore, customized reports that preserve anonymity are generated for students so that they may review and discuss their own performance as a team. We are still learning to apply this tool, and hope to use its results more extensively to help teams in the future.

We have noticed some consistency between ITPmetrics data and our own observations of WDF data. In particular, we hypothesize that WDFs indicating great variability in overall responsibility 
claimed by team members are indications of team dysfunction. We accept ITPmetrics's system of gauging team dysfunction by colour coding based on its internal analyses. In a preliminary review of data from Fall 2019, we compared WDF variability to ITPmetrics reporting. We also compared this data to our own observations of teams during studios and in various team-instructor meetings. We found that many teams that appeared dysfunctional according to ITPmetrics also had significant variability in their WDFs. However, we also noted that both WDFs and ITPmetrics (a) failed to catch all dysfunctional teams, and (b) sometimes identified as dysfunctional teams that seemed in fact to be working well. All we can say with confidence at this time is that both ITPmetrics and WDFs successfully identified many dysfunctional teams and provide a basis for discussion with teams when needed.

In the coming year, having gained experience with ITPmetrics, we expect to gather more detailed information to better compare the tools. There are a number of issues that could impact the effectiveness of both ITPmetrics and the WDF as measures of dysfunctionality, such as social desirability bias (responding according to expectations), peer pressure effects (reluctance to report problems about teammates), and instrument sensitivity (whether false positives are more or less likely than false negatives). These are issues we expect to study in the future.

Another tool we started using in the last few years are very light-weight surprise quizzes during lectures. These quizzes are primarily meant to gauge student attendance in lecture and are only worth $1 \%$ each. Our analysis of the data over time shows clearly that lecture attendance is correlated with final course grade, as shown in Figure 1, below. The figure shows the number of quizzes submitted by a student correlates directly with the student's final grade that for all four years in which we used in-class quizzes. We use this information for two purposes: (a) we show these results to students, in an attempt to motivate them to attend lecture, and (b) we use individual student submissions to help identify dysfunctional teams (i.e., teams who have members who do not attend lecture and are thus unprepared for the design tasks). Of course, we are not sure if this data is indicative of causality or only correlation at this point; we continue to investigate this. 


\section{Class Exercises Submitted vs Final Grade (AGGREGATE) \\ $20192018 \bigcirc 2017 \bigcirc 2016$}

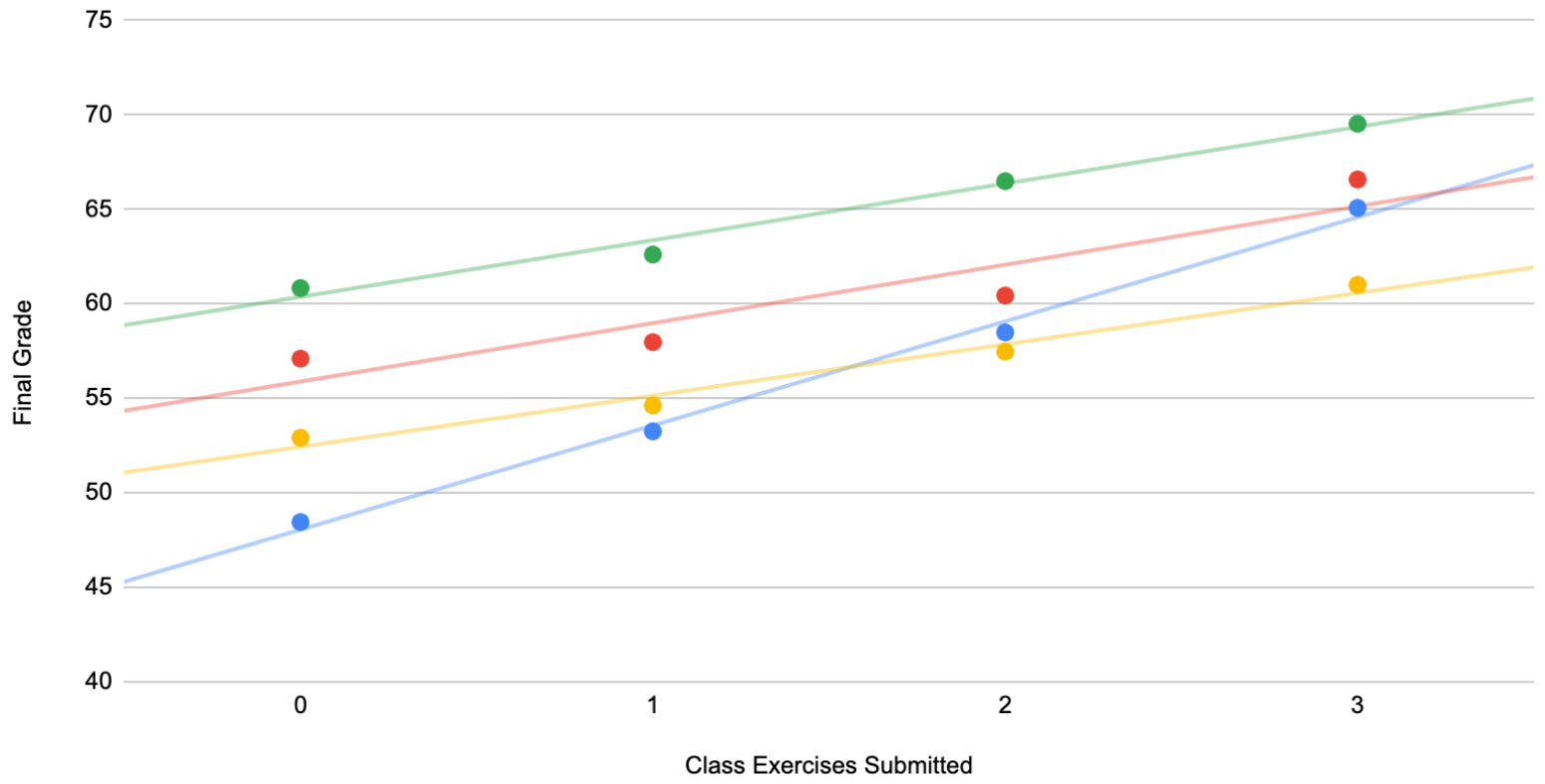

Figure 1: Correlation between class exercises submitted and final course grade, over the four years in which class exercises were used.

We also note an ancillary purpose for Personas to help mitigate team dysfunction. By having each student be responsible for only one Persona, we expect to (a) give students a reasonable way to defend their arguments (e.g., "We need to do $X$ to make sure Samantha [the Student's Persona] is satisfied by our design"), and (b) help redirect possible antagonism by others to the Persona rather than toward the student themselves. We have not yet found a suitable way to assess this particular aspect of our tactic.

Another strategy we have tried to encourage deliberate and reflective team working practices is the use of "team contracts", established at the beginning of each semester, to set a framework for students' behaviours. Every team was provided with a team contract template to complete. The teams are asked to specify their goals, expectations, rules, and consequences when the former are breached. With regards to expectations, the template asks the students to indicate expectations on attendance, participation, communications, conduct, deadlines, and quality. The intention of the team contract was to have the team elicit and articulate their preferred team practices. All team members must sign the contract; each team must submit their contract to the instructors. While the contract is not enforced, it is intended to provide a deliberate framework for each team member to consider their behaviour towards the team and their project.

The contract was also useful in cases of special meetings requested by teams experiencing dysfunction. For these teams the contract could be used as a concrete basis for discussing current problems and re-establishing ground rules for the team to move forwards. Like other teamwork approaches, it is difficult to isolate the impacts of the application of this tool. The 
authors believe it is useful, but are inclined to increase the level of specificity of the template providing a more complete suggested set of behaviours in order to reduce the time spent discussing the contract and move the teams more quickly into their term projects. We note that these pressures would likely be reduced if our course were extended to two semesters in duration.

A further effort to develop teamwork and associated social skills was the deployment of "Live Actor Simulations" with each section of the class. In this effort, three actors were provided with realistic teamwork scenarios and acted as team members for students invited up to the "hot seats" to join the team. In a series of scenes, a form of team dysfunction would emerge (as brought forward by the actors) according to the script of the session. The students were then required to identify and work to resolve the teamwork problem so the project could continue in a good way. Instructors could stop the action at various points to engage the entire class to discuss and analyse both the problem and possible courses of action needed to achieve a good outcome. These sessions were conducted with the support of our institution's Live Actor Simulation program, who provided the teamwork scenario and trained the actors [6]. While these sessions provided a valuable opportunity to discuss critical aspects of teamwork, there remain questions about the efficacy of the approach, the extent of buy-in of the audience who choose not to participate, and the use of an entire week of lab/studio time in a one-semester project. Without a quantitative team function evaluation, and an experimental methodology, the benefits of the live actor approach are difficult to measure. If the incorporation of ITPmetrics (or some other evaluative tool) proves successful, we will try to bring back the Simulations.

\section{Discussions and Future Work}

We have described a number of methods and tools we have used to develop an engineering design cornerstone course that tightly integrates human factors throughout the course. All our efforts were "pre-COVID". It is unclear at this time which of these methods and tools will survive the transition to remote/online teaching and learning.

One aspect of teamwork we have not yet investigated is friction within teams due to cultural differences. Ryerson University - like many other Canadian Universities - becomes more culturally diverse each year. We are aware of some instances of team difficulties arising due to cultural differences between team members, but have not examined the nature and extent of those differences and how one might mitigate the resulting educational friction that arises as a result.

If we were to pass on advice to other design instructors, we would propose the following. We note that though these recommendations arise from our ongoing attempts to embed HF within design, we believe that they would also benefit instructors seeking to embed other design-related issues (e.g., sustainability) into design courses.

- Have all members of the teaching team present at all lectures and labs/studios, and allow for opportunistic interactions with students from other instructors besides the primary for a given lecture or lab/studio. 
- Do not fear having some distinction between course components. The tightest connection in MEC325 between design and HF is in the requirements elicitation and concept evaluation stages, and it is there where we have had the greatest success in integrating the material; we did not recognize this when the course was new.

- Take any opportunity to hire undergraduate research assistants from those students who have done well in your courses to help develop and refine courseware. The undergraduate research assistants that we have hired, having recently completed MEC325 themselves, were well-positioned to feel empathy for future students and tend to produce materials that are more usable by their peers than materials we may produce for them.

- Leverage undergraduate teaching assistants. We have found that they are typically more diligent and enthusiastic for helping students learn than many graduate students may be.

- Ensure consistent and universal terminology, especially if your course combines different fields. Review all notes, slides, and assignments to ensure clear and consistent use of language between the entire teaching team.

- In project work, look for ways to prevent teams from "hiding" their ulterior motives for making project decisions. Teams with ulterior motives may well become dysfunctional, or may be trying to sidestep their academic obligations.

- Keep extensive notes on how students respond to courseware. Take those notes as closely as possible to the moment when observations of student responses are made. Make the time to review those notes as soon as possible after the fact (when your own memories are freshest). Do not just note the observation, but clearly describe problems and list in some detail every possible solution you can envision at the time. The more notes you take at this point, the easier it will be to adjust the course in subsequent months even if your memories of the events fade.

- Focus on showing students how to use information to execute tasks before focusing on how to find that information. Once students understand the significance of information use, we believe they will be more inclined to diligence in searching for it.

\section{Conclusion}

MEC325 has evolved significantly over the last decade. It has improved notwithstanding several false starts and surprisingly failures along the way. Improvement involves considerable experimentation and evolution over time. Since each change requires one year to evaluate, even if only qualitatively, this process can seem slow. Under these circumstances, sharing lessons learned between instructors, via for example CEEA forums and conferences, becomes an important means to move forwards as a discipline.

We hope that our account of our journey will be useful to other instructors. In particular, we hope we've demonstrated how the quality of designs can benefit significantly from the ubiquitous inclusion of HF: when the human is accounted for throughout the development process, designers have more opportunities to attend deeply to user needs, which results in better designs overall. 


\section{References}

[1] D. Clausing, Total Quality Development: A Step-by-step Guide to World Class Concurrent Engineering. ASME Press, 1994.

[2] "A heuristic method for identifying modules for product architectures," Design Studies, vol. 21(1), no. 5-31, Jan. 2000, doi: 10.1016/S0142-694X(99)00003-4.

[3] S. Pugh, Total Design: Integrated Methods for Successful Product Engineering. Addison-Wesley Publishing Company, 1991.

[4] J. Annett, "Hierarchical task analysis," Handbook of cognitive task design, vol. 2, pp. 17-35, 2003, [Online]. Available:

https://books.google.ca/books?hl=en\&lr=\&id=dElPH0ruR-sC\&oi=fnd\&pg=PA17\&dq=\%22hierarchi cal+task+analysis\%22\&ots=EHgRp8-OxC\&sig=NFwUOk31pc6avNJe81VN8Iuwwsw.

[5] S. R. Nicholson, W. Patrick Neumann, M. F. (Frankie) Stewart, and F. A. Salustri, "ADDRESSING DIVERSITY AND GENDER ISSUES IN A CORNERSTONE DESIGN COURSE," Proceedings of the Canadian Engineering Education Association (CEEA), Oct. 2019, doi: 10.24908/pceea.vi0.13740.

[6] W. P. Neumann, R. Rosen, and K. Turner, "Setting the scene for ergonomics-Live actors and action go a long way toward teaching students how to explain human factors," Ind. Eng., vol. 44, no. 3, p. $28,2012$. 